\title{
Micronutrientes e capacidade antioxidante em adolescentes sedentários e corredores
}

\author{
Micronutrients and antioxidant capacity \\ in sedentary adolescents and runners
}

Karla de Jesus Fernandes de OLIVEIRA ${ }^{1}$

Josely Correa KOURY1,2

Carmen Marino DONANGELO'

RE S U M O

\section{Objetivo}

Este estudo objetivou comparar a composição corporal, a ingestão dietética, os índices bioquímicos de micronutrientes antioxidantes e a capacidade antioxidante em adolescentes sedentários $(n=15)$ e corredores (n=18), pós-púberes.

\section{Métodos}

A composição corporal foi aferida por meio das dobras cutâneas, massa corporal total e estatura; a ingestão de micronutrientes foi determinada através de freqüência de consumo alimentar e os indicadores bioquímicos por coleta de sangue em jejum. Em sangue total foram determinados hematócrito e hemoglobina; em plasma, testosterona, $\alpha$-tocoferol, cobre, zinco, e ceruloplasmina; em eritrócitos, fragilidade osmótica, zinco, Cu-Zn superóxido dismutase e metalotioneína.

\section{Resultados}

A capacidade antioxidante, a ingestão dietética e a composição corporal foram similares, exceto o somatório de dobras cutâneas, que foi menor nos corredores $(p<0,05)$. Somente a ingestão de vitamina $E$ foi inferior às recomendações nutricionais. Não houve diferença entre os grupos para a concentração plasmática de $\alpha$-tocoferol, que se apresentou, em média, abaixo do valor de referência. Os níveis plasmáticos de cobre e zinco foram, em média, adequados, sendo os níveis de cobre similares entre os dois grupos e os de zinco maiores nos corredores. Nos sedentários a fragilidade osmótica dos eritrócitos relacionou-se com a metalotioneína ( $r=-0,50 ; p<0,05)$ e com Cu-Zn superóxido dismutase $(r=-0,50 ; p<0,005)$; nos corredores, houve relação entre Cu-Zn superóxido dismutase e zinco nos erirócitos $(r=0,49 ; p<0,005)$.

\footnotetext{
1 Universidade Federal do Rio de Janeiro, Instituto de Química, Departamento de Bioquímica, Laboratório de Bioquímica Nutricional e de Alimentos. Rio de Janeiro, RJ, Brasil.

2 Universidade do Estado do Rio de Janeiro, Instituto de Nutrição, Departamento de Nutrição Básica e Experimental, Centro de Atendimento Nutricional para Atleta. R. São Francisco Xavier, 524, $12^{\circ}$ andar, Sala 12006-D, Maracanã, 20559-900, Rio de Janeiro, RJ, Brasil. Correspondência para/Correspondence to: C.M. DONANGELO. E-mail: <donangel@iq.ufrj.br>.
} 


\section{Conclusão}

Os resultados sugerem que a modalidade estudada - corrida - não alterou a capacidade antioxidante além do próprio crescimento. No entanto, a prática regular de exercício favorece uma composição corporal mais adequada para os adolescentes. Há necessidade de maior atenção quanto ao estado nutricional de $\alpha$-tocoferol em adolescentes.

Termos de indexação: adolescente; cobre; composição corporal; exercício; zinco.

\section{A B S T R A C T}

\section{Objective}

The aim of this study was to compare body composition, biochemical indices of antioxidant micronutrients, intake and nutritional status and antioxidant capacity in post-puberty sedentary adolescents $(n=15)$ and runners ( $n=18$ ).

\section{Methods}

Skin-fold measurements, total body mass and height were used for anthropometric evaluation and a food frequency questionnaire for assessment of micronutrient intake. Biochemical indices measured after an overnight fast included: blood hemoglobin and hematocrit; plasma testosterone, $\alpha$-tocopherol, copper, zinc and ceruloplasmin; and in erythrocytes, osmotic fragility, zinc, Cu-Zn superoxide dismutase and metallothionein.

\section{Results}

Indices of antioxidant capacity, dietary intake, and body composition were not different between the groups, except for the sum of skin-folds that was lower in runners $(p<0.05)$. Most adolescents had vitamin $E$ intakes lower than nutritional recommendations. Plasma levels of copper and zinc were, on average, adequate. Copper levels were similar in both groups and zinc levels were higher in runners. In the sedentary group, erythrocyte osmotic fragility was correlated with metallothionein and Cu-Zn superoxide dismutase ( $r=-0.50$; $p<0.005)$; in runners, erythrocyte superoxide dismutase and zinc were correlated $(r=0.49 ; p<0.005)$.

\section{Conclusion}

Our results suggest that the regular practice of running did not affect the antioxidant capacity of the adolescents studied besides growth. However, this practice seems to favor a more appropriate body composition in adolescents. More attention is necessary on the nutritional status of $\alpha$-tocopherol in adolescents.

Indexing terms: adolescent; copper; body composition; exercise; zinc.

\section{N T R O D U Ç Ã O}

A adolescência é marcada por mudanças hormonais que resultam na maturidade biológica e em alterações da composição corporal' ${ }^{\text {. Dentro }}$ da variabilidade normal, no período da puberdade, adolescentes da mesma idade podem ser classificados em pré-púberes, púberes ou pós-púberes² A maturação e as fases de crescimento são determinantes das necessidades nutricionais em cada momento da adolescência ${ }^{2}$. A demanda nutricional é ainda maior quando a adolescência é associada à prática esportiva, em conseqüência das adaptações fisiológicas que ocorrem no organismo do atleta, tais como hipertrofia das fibras musculares, aumento da síntese de tecido conjuntivo, aumento da capacidade cardiovascular e incremento do conteúdo mineral ósseo, entre outras $^{3}$.

A prática de atividade física regular acarreta aumento do consumo de oxigênio, tendo como conseqüência o incremento da produção de espécies reativas de oxigênio ${ }^{4}$. Essas espécies, altamente instáveis, têm como alvos principais componentes celulares como proteínas, enzimas, lipídeos, ácido desoxirribonucléico (DNA) e ácido ribonucléico (RNA), e sua ação ocasiona lesões na célula, alterando sua integridade e, conseqüentemente, sua funcionalidade ${ }^{5}$. Apesar de favorecer o processo oxidativo, a atividade física também estimula a capacidade antioxidante ${ }^{6}$. 
Os antioxidantes de defesa celular neutralizam a proliferação ou protegem a membrana celular da ação lesiva das espécies reativas de oxigênio, podendo ser intra ou extracelulares, enzimáticos ou não enzimáticos. Vários nutrientes da dieta são importantes para o adequado funcionamento dos sistemas antioxidantes. Entre eles destacam-se o $\alpha$-tocoferol, protetor de membranas celulares, e os minerais zinco e cobre, componentes de metaloproteínas, tais como superóxido dismutase, ceruloplasmina e metalotioneína ${ }^{7,8}$.

A maior parte das pesquisas feitas na área de nutrição esportiva e capacidade antioxidante tem sido realizada em adultos ${ }^{9,10}$. A resposta da capacidade antioxidante ao exercício em adolescentes, apesar de ser, possivelmente, semelhante em termos qualitativos à do adulto, poderia também ser influenciada pelo rápido crescimento e desenvolvimento próprios da adolescência.

Não foram encontrados estudos que relacionassem níveis de micronutrientes e capacidade antioxidante em adolescentes fisicamente ativos. Este estudo objetivou comparar a composição corporal, a ingestão dietética, os índices bioquímicos de micronutrientes antioxidantes ( $\alpha$-tocoferol, zinco e cobre) e a capacidade antioxidante plasmática (ceruloplasmina) e eritrocítica (Cu-Zn superóxido dismutase, metalotioneína, zinco) em dois grupos de adolescentes, sedentários e praticantes de corrida como atividade física regular.

\section{MÉTODOS}

Foram estudados 33 adolescentes, sendo 15 sedentários - sem atividade física regular e intensa - e 18 praticantes de corrida (100-400m) atividade física regular e intensa -, do gênero masculino, com idades entre 14 e 18 anos, saudáveis e sem uso recente de medicamentos e/ou suplementos nutricionais.

Os corredores foram selecionados ao acaso, pela Federação de Atletismo do Rio de
Janeiro (FARJ), entre aqueles que mais se destacaram em diferentes competições. Os próprios corredores indicaram os adolescentes sedentários entre seu círculo de convívio, fato que garantiu a homogeneidade dos grupos em relação ao hábito alimentar.

Todos os voluntários participaram, após consentimento expresso e autorização dos pais, que receberam documento esclarecendo os objetivos do estudo. O protocolo do estudo foi previamente aprovado pelo Comité de Ética do Hospital Universitário Pedro Ernesto.

A coleta de dados envolveu aplicação de questionário de dados gerais, questionário de freqüência de consumo alimentar, aferições antropométricas e coleta de amostra de sangue para as análises bioquímicas.

Os dados de ingestão dietética habitual foram obtidos por meio de questionário de freqüência de consumo alimentar, visando as principais fontes dos micronutrientes de interesse. A quantificaçãao da ingestão diária dos nutrientes foi feita com o auxílio do programa computacional The Food Processor Nutrition Analysis Software ("Version 7.20. Copyright 1998 - ESHA Research"), adaptado para alimentos brasileiros.

A avaliação antropométrica foi realizada no dia que antecedeu a coleta de sangue, sob supervisão de um antropometrista qualificado, envolvendo medição de massa corporal total e estatura (aferidos com balança antropométrica Filizola) e de dobras cutâneas (tríceps, bíceps, supra-ilíaca, subescapular, axilar, abdominal e coxa média), aferidas com espessímetro de Lange, conforme descrito por Lohman ${ }^{11}$. Foi calculado o somatório das sete dobras cutâneas para quantificar o depósito de gordura subcutânea corporal.

Para avaliação bioquímica, foi coletado sangue venoso pela manhã (8h), após jejum noturno de $12 \mathrm{~h}$, em ambos os grupos. Os adolescentes praticantes de corrida estavam sem treinar por 24 horas. O sangue foi transferido para dois tubos de ensaio, um contendo heparina como anticoagu- 
lante (30U/tubo) e outro sem anticoagulante para obtenção de soro. No sangue total foram analisados hematócrito e hemoglobina por metodologias convencionais. Para determinar o período de maturação sexual foi determinada a concentração plasmática de testosterona, por radioimunoensaio (Diagnostic Products Corporation - Coat-A-Count Total Testosterone, USA).

No plasma foram analisados: cobre e zinco, por espectrometria de absorção atômica ${ }^{9}$, ceruloplasmina, determinada pela atividade oxidase, de acordo com o método de Sunderman \& Nomoto $^{12}$ e $\alpha$-tocoferol, por cromatografia líquida de alta eficiência, segundo o método de Hess et al. ${ }^{13}$, corrigido pela concentração de colesterol determinada por kit comercial ("Doles" - Colesterol 250, Brasil). Houve necessidade de correção pelo colesterol, uma vez que é reconhecido que a composição lipídica do plasma, principalmente a de colesterol, é um determinante importante das concentrações de $\alpha$-tocoferol no plasma ${ }^{7,14}$. Nos eritrócitos foram determinados: fragilidade osmótica, a partir da adaptação do método de O'Dell et al. ${ }^{15}$, que se baseia na determinação do percentual de hemólise de eritrócitos incubados in vitro em meio hipotônico; a atividade da cobre-zinco superóxido dismutase, baseada na autoxidação do pirogalol'16; metalotioneína, medida por sua afinidade pelo cádmio ${ }^{17}$ e zinco, por espectrometria de absorção atômica9 .

As análises estatísticas foram realizadas com auxílio do programa computacional SCGPlus Version 7.0 (Statistical Graphics Corporation Plusware, USA). As mesmas incluíram: análise descritiva, análise de correlação em matriz de Pearson (paramétrica) e teste - " $\mathrm{t}$ " de Student para comparação entre os grupos. Valores de $p<0,05$ foram considerados significantes.

\section{RESULTA DOS}

Os adolescentes estudados apresentavam idade média de 16, com desvio-padrão (DP) de 1,2 anos (sedentários- $15,7, \mathrm{DP}=1,5$ anos e corredores- $16,2, \mathrm{DP}=0,9$ anos). A maturação biológica dos voluntários sedentários e corredores foi avaliada a partir dos níveis de testosterona plasmática $(19, \mathrm{DP}=7 \mathrm{nmol} / \mathrm{L}$ e $21, \mathrm{DP}=5 \mathrm{nmol} / \mathrm{L}$, respectivamente), sendo todos classificados como pós-púberes (testosterona plasmática $>5,21 \mathrm{nmol} / \mathrm{L})^{18}$. A concentração de testosterona plasmática foi semelhante entre os grupos e não se relacionou com nenhum indicador estudado.

Os corredores relataram, em média, 5 $(\mathrm{DP}=1,3)$ anos de prática esportiva na sua modalidade, com treinos de, aproximadamente, 3 horas diárias.

Em geral, os adolescentes sedentários e corredores apresentaram composição corporal semelhante, embora o somatório das sete dobras cutâneas tenha sido maior nos sedentários (Tabela1). Entre as dobras avaliadas, a abdominal e a da coxa foram maiores nos sedentários (abdominal $13,9, D P=6,4 \mathrm{~mm}$ e coxa $14,7, \mathrm{DP}=3,6 \mathrm{~mm}$ ) do que nos corredores (abdominal 10,5, DP=2,4mm e coxa $11,4, \mathrm{DP}=3,0 \mathrm{~mm})(p<0,05)$.

No grupo dos corredores, a massa livre em gordura se relacionou positivamente com a massa corporal total $(r=0,970 ; p<0,001)$, sugerindo a influência do exercício sobre a composição corporal.

A ingestão dos nutrientes nos grupos estudados foi semelhante (Tabela 2). Quando comparados aos valores médios de necessidade estimados para esta faixa etária e gênero, 100\% dos adolescentes apresentaram ingestão de

Tabela 1. Características antropométricas dos adolescentes. Rio de Janeiro, 2004.

\begin{tabular}{|c|c|c|c|c|c|c|}
\hline & \multicolumn{3}{|c|}{ Sedentários $(n=15)$} & \multicolumn{3}{|c|}{ Corredores $(n=18)$} \\
\hline & M & DP & Mediana & M & $\mathrm{DP}$ & Mediana \\
\hline Estatura (m) & 1,74 & 0,1 & 1,76 & 1,74 & 0,1 & 1,72 \\
\hline MCT (kg) & 66,3 & 17,0 & 59,4 & 63,40 & 4,6 & 63,40 \\
\hline MLG (kg) & 57,0 & 8,8 & 54,0 & 58,00 & 4,5 & 59,00 \\
\hline $\begin{array}{l}\text { Somatório de } \\
\text { dobras cutânea }\end{array}$ & $\begin{array}{l}85,7^{\mathrm{a}} \\
(\mathrm{mm})\end{array}$ & 24,9 & 78,0 & $70,00^{\mathbf{b}}$ & 11,9 & 70,00 \\
\hline
\end{tabular}

Resultados expressos como (M) média; (DP) desvio-padrão; Mediana; MCT: massa corporal total; MLG: massa livre em gordura, somatório das sete dobras cutâneas. Valores com letras diferentes no supra-índice na mesma linha são significantemente diferentes $(p<0,05)$. 
vitamina $\mathrm{E}$ inferior, $73 \%$ ingeriam cobre acima e $55 \%$ consumiam zinco no limite do requerimento. A ingestão dietética não influenciou a composição corporal nem os indicadores bioquímicos avaliados.

O hematócrito e a concentração de hemoglobina no sangue foram, em média, adequados ${ }^{19}$ e similares (hemoglobina-sedentários 13,2, $\mathrm{DP}=1,5 \mathrm{~g} / \mathrm{dL}$ e corredores $13,8, \mathrm{DP}=1,5$; hematócrito- sedentários $45,3, \mathrm{DP}=4,3 \%$ e corredores $47,1, \mathrm{DP}=3,1 \%$ ).

Não foram encontradas diferenças significantes nos indicadores relacionados aos micronutrientes e a capacidade antioxidante. Somente o nível de zinco plasmático foi significantemente maior nos adolescentes corredores, quando comparado aos sedentários $(p<0,05)$. A fragilidade osmótica dos eritrócitos foi semelhante entre os grupos, com coeficiente de variação de 30\% para os atletas e 11\% para os sedentários (Tabela 3).

Tabela 2. Ingestão habitual de micronutrientes antioxidantes. Rio de Janeiro, 2004.

\begin{tabular}{|c|c|c|c|c|c|c|c|}
\hline & \multicolumn{3}{|c|}{$\begin{array}{l}\text { Sedentários } \\
\quad(n=15)\end{array}$} & \multicolumn{3}{|c|}{$\begin{array}{c}\text { Corredores } \\
(n=18)\end{array}$} & \multirow{2}{*}{$\begin{array}{c}\text { Valores de } \\
\text { Referêcia } \\
(\text { EAR })^{*}\end{array}$} \\
\hline & $\mathrm{M}$ & $\mathrm{DP}$ & Med & $M$ & $\mathrm{DP}$ & Med & \\
\hline Vitamina $\mathrm{E}(\mathrm{mg} / \mathrm{dia})$ & 4,2 & 0,2 & 4,3 & 4,5 & 0,2 & 4,2 & $12 \mathrm{mg} / \mathrm{dia}$ \\
\hline Zinco (mg/dia) & 8,5 & 0,8 & 7,8 & 8,9 & 1,0 & 8,1 & $8,5 \mathrm{mg} / \mathrm{dia}$ \\
\hline Cobre (mg/dia) & 1,2 & 0,2 & 1,1 & 1,0 & 0,4 & 0,9 & $0,7 \mathrm{mg} / \mathrm{dia}$ \\
\hline
\end{tabular}

M: média; DP: desvio-padrão; Med: mediana; EAR: Estimated Average Requeriment ${ }^{23,24}$.
Foram encontradas várias relações entre os indicadores bioquímicos, porém somente no grupo dos sedentários, a fragilidade osmótica dos eritrócitos apresentou relação negativa com a metalotioneína $(r=-0,50 ; p<0,005)$ e também com a atividade da Cu-Zn superóxido dismutase $(r=-0,50 ; p<0,005)$; nos corredores, foi observada relação positiva da atividade $\mathrm{Cu}-Z n$ superóxido dismutase com zinco eritrocítico $(r=0,49 ; p<0,005)$.

\section{I S C U S S Ã O}

Estudos descrevem que estados fisiológicos especiais, como a adolescência, apresentam aumento de danos oxidativos em sistemas biológicos, decorrentes do intenso crescimento tecidual e de alterações hormonais característicos desta fase $^{20}$. No caso de adolescentes atletas, a atividade física associada poderia favorecer o estímulo da capacidade antioxidante, bem como promover alterações favoráveis à composição corporal. Além disso, um adequado aporte de micronutrientes, tais como cobre, zinco e vitamina E é capaz de manter a homeostase influenciada pelo rápido crescimento e pela prática de atividade física intens $\mathrm{a}^{4,8}$.

A classificação dos adolescentes em pré-púberes, púberes ou pós-púberes, pode ser realizada a partir da concentração de hormônios sexuais ${ }^{2}$. Cada fase do desenvolvimento pode

Tabela 3. Indicadores bioquímicos relacionados a micronutrientes e à capacidade antioxidante. Rio de Janeiro, 2004.

\begin{tabular}{|c|c|c|c|c|c|c|}
\hline & \multicolumn{3}{|c|}{ Sedentários $(n=15)$} & \multicolumn{3}{|c|}{ Corredores $(n=18)$} \\
\hline & $\mathrm{M}$ & DP & Mediana & M & DP & Mediana \\
\hline Zinco plasmático ( $\mu \mathrm{mol} / \mathrm{L})$ & $19,00^{\mathrm{a}}$ & 2,40 & 19,90 & $22,00^{\mathbf{b}}$ & 4,40 & 22,50 \\
\hline Zinco eritrocítico $(\mu \mathrm{g} / \mathrm{gHb})$ & 20,90 & 0,02 & 20,90 & 20,90 & 0,01 & 20,90 \\
\hline Metalotioneína em eritrócitos ( $\mathrm{nmol} / \mathrm{gHb}$ ) & 4,70 & 1,30 & 4,10 & 4,40 & 1,40 & 4,70 \\
\hline Fragilidade osmótica de eritrócitos (\%) & 53,30 & 6,10 & 60,00 & 57,30 & 17,20 & 56,50 \\
\hline Cobre plasmático $(\mu \mathrm{mol} / \mathrm{L})$ & 16,00 & 0,05 & 15,90 & 16,00 & 0,04 & 15,90 \\
\hline Ceruloplasmina em plasma (mg/dL) & 36,30 & 7,00 & 35,50 & 38,40 & 10,20 & 37,00 \\
\hline Cobre-Zinco superóxido dismutase em eritrócitos (U/mgHb) & 1,10 & 0,20 & 1,10 & 1,20 & 0,20 & 1,20 \\
\hline$\alpha$-Tocoferol plasmático* $(\mu \mathrm{mol} / \mathrm{mmol}$ colesterol) & 0,39 & 0,11 & 0,41 & 0,32 & 0,20 & 0,35 \\
\hline$\alpha$-Tocoferol plasmático $(\mu \mathrm{mol} / \mathrm{L})$ & 11,70 & 2,70 & 12,6 & 10,10 & 6,50 & 11,70 \\
\hline
\end{tabular}

Resultados expressos como (M) média; (DP) desvio-padrão; mediana. Valores com letras diferentes no supra-índice na mesma linha são significantemente diferentes $(p<0,05)$; *corrigido pelo colesterol plasmático. 
acarretar diferentes respostas metabólicas, características em função das alterações hormonais².

Neste estudo, a concentração plasmática de testosterona demonstrou que os adolescentes estavam no período pós-púbere. Possivelmente, por este motivo não foram encontradas correlações entre os níveis de testosterona e os indicadores estudados, sugerindo que nesta fase o decréscimo na velocidade do crescimento não interfere diretamente sobre os indicadores bioquímicos e antropométricos avaliados.

A atividade física e o hábito alimentar são capazes de alterar a composição corporal². O grau de alteração gerada pelo exercício depende do tipo, da intensidade e da freqüência, além da forma de obtenção de energia (aeróbia ou anaeróbia). Atletas de modalidades basicamente aeróbias tendem a ter menor acúmulo de gordura corporal, devido à maior mobilização de ácidos graxos ${ }^{21}$.

Neste estudo, observou-se como um dos maiores sítios de depósito de gordura corporal a região abdominal, sendo esse acúmulo maior nos sedentários do que nos corredores, mostrando-se consistente com a mobilização do tecido adiposo decorrente da modalidade esportiva avaliada. O maior acúmulo de gordura na região abdominal, em indivíduos sedentários, pode indicar uma provável predisposição ao surgimento de doenças cardiovasculares ${ }^{22}$, sendo importante a prática de atividade física regular por adolescentes na prevenção de futuras alterações cardiovasculares.

A ingestão de micronutrientes por atletas vem sendo bastante estudada, apesar disso não foi encontrado nenhum estudo que relatasse a ingestão de vitamina $\mathrm{E}$ em atletas adolescentes. A ingestão de zinco e cobre tem sido avaliada em estudantes praticantes de atividade física regular, principalmente natação ${ }^{10}$

No presente estudo, considerando todos os adolescentes, foi observado que a ingestão habitual de cobre encontrava-se elevada, quando comparada ao valor de referência ${ }^{23}$, provavelmente, pelo elevado consumo de carnes e leguminosas. A ingestão de zinco estava no limite de adequação ${ }^{23}$ para os adolescentes que relataram menor consumo de carne vermelha. Todos os adolescentes apresentaram consumo de vitamina $\mathrm{E}$ inadequado (40\% de inadequação), quando comparado aos valores de referência ${ }^{24}$. A elevada freqüência de adolescentes com ingestão inferior ao requerimento médio estimado para vitamina E poderia ser, em parte, explicada pelo sub-relato de óleos e gorduras adicionados às preparações.

O exercício físico regular exerce alteração na homeostase de diferentes micronutrientes ${ }^{25}$. 0 zinco é um elemento traço que participa de mais de 300 enzimas, além disso, é considerado um importante antioxidante, por manter a estabilidade das membranas celulares e compor metaloproteínas antioxidantes ${ }^{26}$. O cobre desempenha várias funções importantes ligadas ao exercício, entre elas destacam-se sua participação no metabolismo energético, como sítio ativo da ceruloplasmina e da síntese de hemoglobina e mioglobina ${ }^{8}$. O $\alpha$-tocoferol é um dos isômeros que compõem a vitamina $E^{7}$. Resultados de estudos com $\alpha$-tocoferol em atletas são conflitantes, mas é reconhecido que este micronutriente é imprescindível para manutenção da capacidade antioxidante, pois constitui um potente seqüestrador de radicais peroxila, e protege as cadeias de ácidos graxos insaturados dos fosfolipídios, componentes de membranas biológicas e os das lipoproteínas plasmáticas ${ }^{7}$.

Os adolescentes deste estudo apresentaram níveis de zinco plasmático ( $\left.>11,5 \mu \mathrm{mol} / \mathrm{L}^{23}\right)$ adequados, porém os corredores apresentaram maior concentração plasmática deste mineral. Este resultado pode estar relacionado com a maior ocorrência de micro-lesões musculares associadas ao impacto e explosão característicos da corrida, induzindo assim maior liberação de zinco do tecido muscular, que sofreu injúria, para o plasma ${ }^{27}$. Semelhantemente a estes resultados, Fogelholm et al. ${ }^{28}$ encontraram maior concentração de zinco plasmático em atletas adolescentes de diferentes modalidades esportivas, quando comparados ao grupo controle. 
Para ambos os grupos de adolescentes, os níveis plasmáticos de cobre foram similares e adequados $\left(>10 \mu \mathrm{mol} / \mathrm{L}^{23}\right)$, sugerindo que a corrida não exerceu influência sobre a homeostase do cobre. Estes resultados foram semelhantes a estudos realizados com nadadores da mesma faixa etária ${ }^{10}$.

Não foram encontrados estudos sobre a concentração plasmática de $\alpha$-tocoferol em adolescentes fisicamente ativos. Neste estudo, não foi observada diferença significante entre os níveis de $\alpha$-tocoferol plasmático nos grupos estudados, porém $52 \%$ de todos os adolescentes apresentaram valores inferiores àquele considerado adequado $^{24}(12 \mu \mathrm{mol} / \mathrm{L})$. Apesar de não encontrar relação estatística, possivelmente, em função do tamanho amostral, este resultado pode ser devido à reduzida ingestão de vitamina $\mathrm{E}$ observada em todos os adolescentes.

Os eritrócitos podem sofrer alterações funcionais e estruturais decorrentes da atividade física intensa. Indivíduos não treinados têm demonstrado menor índice de deformabilidade dos eritrócitos e redução na susceptibilidade à hemólise peroxidativa ${ }^{29}$, do que quando comparados a atletas. A deformabilidade dos eritrócitos e a susceptibilidade à hemólise são influenciadas pela fluidez da membrana celular, pela razão área da superfície: volume total e pela composição da membrana celular e do citoesqueleto ${ }^{29}$.

Neste estudo, a fragilidade osmótica dos eritrócitos foi similar nos dois grupos de adolescentes. Possivelmente, isso indica que não houve diferenças estruturais e funcionais nos eritrócitos dos adolescentes estudados, e que os dois grupos, em média, possuem a mesma capacidade antioxidante, protegendo os eritrócitos de forma semeIhante, embora, o coeficiente de variação tenha sido maior nos atletas do que nos sedentários.

Indicadores de capacidade antioxidante não têm sido mensurados em atletas adolescentes. Em um estudo realizado com ratos, foi observado que o exercício crônico induz a uma adaptação nos níveis de metalotioneína de forma bastante específica para cada tipo de tecido ${ }^{4}$. Esse fato ocorre em função da proteção celular contra a oxidação causada pelos radicais livres e outros fatores ${ }^{4}$. A metalotioneína é uma proteína de baixo peso molecular $(6-7 \mathrm{kDa})$ que contém 20 grupos cisteinil, que liga 5-7 átomos de zinco por molécula. O efeito protetor está relacionado à redução dos resíduos de cisteinil, que captam os radicais peroxila e hidroxila ${ }^{23}$. Somente um estudo avaliou a concentração de metalotioneína em eritrócitos de atletas, segundo o qual foi encontrada elevada concentração em atletas de modalidades de elevado impacto como triatlo e corrida ${ }^{9}$. No presente estudo, não se verificou diferença significante na concentração de metalotioneína entre adolescentes corredores e sedentários. Este resultado sugere que a intensidade do exercício realizado pelos adolescentes não foi suficiente para alterar a síntese de metalotioneína, além do estímulo próprio do crescimento.

A ceruloplasmina pode ser utilizada como indicador de capacidade antioxidante, devido à inibição da reação de Fenton atuando como ferroxidase e captando íons superóxido e outras espécies reativas no plasma ${ }^{8}$. Por apresentar este perfil bioquímico, a ceruloplasmina plasmática tem sido utilizada para avaliar o estado antioxidante. Neste estudo, os níveis de ceruloplasmina no plasma não foram afetados pelo exercício. Porém, em estudo realizado com adultos, a ceruloplasmina se mostrou importante antioxidante para corredores de elite ${ }^{9}$.

A enzima citoplasmática cobre-zinco superóxido dismutase é responsável pela dismutação dos ânions superóxido a oxigênio e peróxido de hidrogênio ${ }^{26}$. A atividade da superóxido dismutase parece aumentar como resultado do aumento da produção do radical superóxido durante o exercício ${ }^{6}$. A atividade da superóxido dismutase, em estudos com humanos fisicamente ativos, é controversa ${ }^{30}$, uma vez que há influência do efeito, do tipo de exercício e da duração do treinamento ${ }^{21}$. A discrepância entre os resultados pode ser explicada pelos diferentes métodos analíticos usados, por diferenças na freqüência e intensidade de treinamentos testados. No pre- 
sente estudo, diferentemente do esperado, não se observou diferença significante na concentração de Cu-Zn superóxido dismutase, apesar da atividade física regular desenvolvida.

Foram identificadas várias relações entre os indicadores bioquímicos avaliados. A relação negativa entre fragilidade osmótica dos eritrócitos com metalotioneína e com Cu-Zn superóxido dismutase, no grupo dos adolescentes sedentários, sugere mecanismos de proteção aos danos oxidativos decorrentes do crescimento característico dessa fase da adolescência ${ }^{20}$. Nos corredores, observou-se que Cu-Zn superóxido dismutase apresentou relação positiva com o zinco dos eritrócitos, sendo esta associação consistente com uma maior demanda de zinco para a proteção de membranas de eritrócitos, frente ao aumento do estresse oxidativo causado pela prática esportiva e crescimento.

Em conclusão, avaliando estes resultados em conjunto, constata-se que, possivelmente, a reduzida ingestão de vitamina $\mathrm{E}$ afetou os níveis plasmáticos dessa vitamina em ambos os grupos de adolescentes, tornando-se importante o acompanhamento nutricional para evitar que a ingestão inadequada atinja o delicado equilíbrio desse nutriente. A modalidade esportiva estudada (corrida de curta distância) não exerceu influência sobre os indicadores bioquímicos e de capacidade antioxidante nos adolescentes avaliados, provavelmente, por não ser realizada com intensidade e/ou duração que promova alterações oxidativas maiores do que as do próprio crescimento típico da adolescência. No entanto, a prática desta modalidade esportiva favorece a composição corporal mais adequada em adolescentes, visto que a mesma se associou a uma menor dobra cutânea abdominal, reconhecida como prejudicial à saúde.

\section{REFERÊ N CIAS}

1. Mascarenhas MR, Zemelb S, Tershakovec AM, Stallings VA. Adolescence. Present knowledge in nutrition. Washington (DC): ILSI; 2001. p.329-43.
2. Rogol AD, Clark PA, Roemmich JN. Growth and pubertal development in children and adolescents: effects of diet and physical activity. Am J Clin Nutr. 2000; 72(2 Suppl):521S-8S.

3. Rockett HRH, Coldiz G. Assessing diets of children and adolescents. Am J Clin Nutr. 1997; 65(Suppl): 1116S-22S.

4. Ji LL. Antioxidants and oxidative stress in exercise. Soc Exp Biol Med. 1999; 222(3):283-92.

5. Cheeseman $\mathrm{KH}$, Slater TF. Free radicals in medicine. Br Med Bull. 1993; 49(3):125-30.

6. Rádak Z. Free radicals in exercise and aging, Champaign, IL: Human Kinetics; 2000. p.343.

7. Atalay M, Laaksonene DE, Khanna S, Kaliste S, Hänninen O, En CK. Vitamin E regulates changes in tissue antioxidants induced by fish oil and acute exercise. Med Sci Sports Exerc. 2000; 32(3):601-7

8. Powers KS, Hamilton NK. Antioxidants and exercise. Clin Sports Med. 1999; 18(3):525-36.

9. Koury JC, Oliveira AV, Portella ES, Oliveira CF, Lopes GC, Donangelo CM. Zinc and copper biochemical indices of antioxidant status in elite athletes of different modalities. Int I Sport Nutr Exerc Metabol. 2004; 14(3):358-72

10. Lukaski HC, Siders WA, Hoverson BS, Gallagher SK. Iron, Copper, Magnesium and Zinc Status as Predictors of Swimming Performance. Int J Sports Med. 1996; 17(7):535-40.

11. Lohman TG, Roche AF, Martorell R. Anthropometric standardization reference manual. Champaign, II: Human Kinetics; 1988.

12. Sunderman FW, Nomoto S. Measurement of human serum ceruloplasmina by its $p$-phenylenediamine oxidase activity. Clin Chem. 1970; 16(11):903-10.

13. Hess D, Keller HE, Oberlin B, Bonfanti R, Schuep W. Simultaneous determination of retinol, tocopherols, carotenes and lycopene in plasma by means of high-performance liquid-chromatography on reversed phase. Int J Vit Nutr Res. 1991; 61(3): 232-38.

14. Neuhouser $M L$, Rock $C L$, Eldridge $A L$, Kriscal $A R$, Patterson RE, Cooper DA, et al. Serum Concentrations of Retinol, $\alpha$-tocopherol and the Carotenoids are Influenced by Diet, Race and Obesity in a Sample of Healthy Adolescents. Am Soc Nutr Sci. 2001; 131(8):1284-91.

15. O'Dell BL, Browining JD, Reeves GP. Zinc deficiency increases the osmotic fragility of rat erythrocytes. Am Inst Nutr. 1987; 117(11):1883-89.

16. Marklund S, Marklund G. Involvement of the superoxide anion radical in the auto oxidation of pyrogallol and a convenient assay for superoxide dismutase. Eur J Bioch. 1974; 47(3):469-74 
17. Zapata CLV, Simões TMR, Donangelo CM. Erythrocyte metallotionein in relation to other biochemical zinc indices in pregnant and nonpregnat woman. Biol Trace Elem Res. 1997; 57(2): 115-23.

18. Garcia-Mayor RV, Andrade MA, Rios M, Lage M, Dieguez C, Casanueva FF. Serum leptine levels in normal children: relationship to age, gender, body mass index, pituttary-gonadal hormones, and pubertal stage. J Clin Endocrinol Metab. 1997; 82(9):2849-55.

19. Sacher RA, McPherson RA. Interpretação clínica dos exames laboratoriais. São Paulo: Manole; 2002. p. 1060.

20. Granot E, Kohen R. Oxidative stress in childhood: in health and disease states. Clin Nutr. 2004; 24(1):3 -11.

21. Heyward VH, Stolarczyk LM, Roemmich JN. Composição corporal e atletas: avaliação da composição corporal aplicada. 2000. p.159-71.

22. Hardman AE. Interaction of physical activity and diet: implications for lipoprotein metabolism. Public Health Nutr. 1999; 2(3a):369-76.

23. Food and Nutrition Board. Dietary reference intakes for vitamin A; vitamin K; arsenic; boron; chromium; copper; iodine; iron; manganese; molybdenum; nickel; silicon; vanadium and zinc. Washington (DC): National Academic Press; 2001.

24. Food and Nutrition Board. Dietary reference intakes for vitamin C, vitamin E, selenium, and carotenoids.
Dietary reference intakes: use in dietary assessment. Food and nutrition board. Washington (DC): National Academy Press; 2000.

25. Smith JA, Kolbuch-Braddon M, Gillan I, Telford RD, Weidmann MJ. Changes in the susceptibility of red blood cell to oxidative and osmotic stress following submaximal exercise. Eur J Appl Physiol. 1995; 70(5):427-36.

26. Dibley MJ. Zinc. In: Bowman BA, Russel RM. Present knowledge in nutrition. Washington (DC); 2001. p.329-43.

27. Cordova A, Navas FJ. Effect of training on zinc metabolism. Changes in serum and sweat zinc concentration in sports men. Ann Nutr Metab. 1998; 42(5):274-82.

28. Fogelholm M. Indicators of vitamin and mineral status in athletes' blood: a review. Int J Sport Nutr. 1995; 5(4):267-84.

29. Beutler $E$, Kuhl W, West C. The osmotic fragility of erythrocytes after prolonged liquid storage and after reinfusion. Blood. 1982; 59(6):1141-7.

30. Mena P, Maynar M, Gutierrez JM, Maynar J, Timon J, Campillo JE. Erythrocyte free radical scavenger enzymes in bicycle professional racers. Adaptation to training. Int J Sports Med. 1991; 12(6):565-6.

Recebido em: 25/4/2005

Versão final reapresentada em: 13/2/2006

Aprovado em: 17/10/2006 
